\title{
OPTIMAL CONSTANTS FOR A MIXED LITTLEWOOD TYPE INEQUALITY
}

\author{
TONY NOGUEIRA, DANIEL NÚÑEZ-ALARCÓN, AND DANIEL PELLEGRINO
}

Abstract. For $p \in[2, \infty]$ a mixed Littlewood-type inequality asserts that there is a constant $C_{(m), p} \geq 1$ such that

$$
\left(\sum_{i_{1}=1}^{\infty}\left(\sum_{i_{2}, \ldots, i_{m}=1}^{\infty}\left|T\left(e_{i_{1}}, \ldots, e_{i_{m}}\right)\right|^{2}\right)^{\frac{1}{2} \frac{p}{p-1}}\right)^{\frac{p-1}{p}} \leq C_{(m), p}\|T\|
$$

for all continuous real-valued $m$-linear forms on $\ell_{p} \times c_{0} \times \cdots \times c_{0}$ (when $p=\infty, \ell_{p}$ is replaced by $c_{0}$ ). We prove that for $p>2.18006$ the optimal constants $C_{(m), p}$ are $\left(2^{\frac{1}{2}-\frac{1}{p}}\right)^{m-1}$. When $p=\infty$, we recover the best constants of the mixed $\left(\ell_{1}, \ell_{2}\right)$-Littlewood inequality.

\section{INTRODUCTION}

The Hardy-Littlewood inequality $([17,1934)$ is a continuation of famous works of Littlewood ([18, 1930) and Bohnenblust and Hille $([9], 1931)$ and can be stated as follows:

- [17. Theorems 2 and 4] If $p, q \geq 2$ are such that

$$
\frac{1}{2}<\frac{1}{p}+\frac{1}{q}<1
$$

then there is a constant $C_{p, q} \geq 1$ such that

$$
\left(\sum_{j, k=1}^{\infty}\left|A\left(e_{j}, e_{k}\right)\right|^{\frac{p q}{p q-p-q}}\right)^{\frac{p q-q-p}{p q}} \leq C_{p, q}\|A\|
$$

for all continuous bilinear forms $A: \ell_{p} \times \ell_{q} \rightarrow \mathbb{R}\left(\right.$ or $\mathbb{C}$ ). Moreover the exponent $\frac{p q}{p q-p-q}$ is optimal.

- [17. Theorems 1 and 4] If $p, q \geq 2$ are such that

$$
\frac{1}{p}+\frac{1}{q} \leq \frac{1}{2}
$$

then there is a constant $C_{p, q} \geq 1$ such that

$$
\left(\sum_{j, k=1}^{\infty}\left|A\left(e_{j}, e_{k}\right)\right|^{\frac{4 p q}{3 p q-2 p-2 q}}\right)^{\frac{3 p q-2 p-2 q}{4 p q}} \leq C_{p, q}\|A\|
$$

for all continuous bilinear forms $A: \ell_{p} \times \ell_{q} \rightarrow \mathbb{R}($ or $\mathbb{C})$. Moreover the exponent $\frac{4 p q}{3 p q-2 p-2 q}$ is optimal.

Above and henceforth, as usual in this field, when $p$ and/or $q$ is infinity, we consider $c_{0}$ instead of $\ell_{p}$ and/or $\ell_{q}$.

As mentioned in [20, Theorem 1] an unified version of the above two results of Hardy and Littlewood asserts that there is a constant $C_{p, q} \geq 1$ such that

$$
\left(\sum_{j=1}^{\infty}\left(\sum_{k=1}^{\infty}\left|A\left(e_{j}, e_{k}\right)\right|^{2}\right)^{\frac{\lambda}{2}} \leq C_{p, q}\|A\|\right.
$$

with $\lambda=\frac{p q}{p q-p-q}$, for all continuous bilinear forms $A: \ell_{p} \times \ell_{q} \rightarrow \mathbb{R}$ (in fact, in [20, Theorem 1] just the complex case is considered, but for a general approach including the real case we refer to [11] moreover the exponents are optimal). The recent years witnessed an increasing interest in the study of summability of multilinear operators

2010 Mathematics Subject Classification. 11Y60, 46G25.

Key words and phrases. Absolutely summing operators; Hardy-Littlewood inequality; Bohnenblust-Hille inequality; Multiple summing operators.

T. Nogueira was supported by Capes, D. Núñez-Alarcón was supported by Capes, Grant 000785/2015-06, and D. Pellegrino was supported by CNPq. 
(see, for instance, 10, 23, 24) and in estimating constants of the multilinear and polynomial Hardy-Littlewood and related inequalities (see [2, 3, 4, 6, 14, 15, 26]). Perhaps the main motivations are potential applications (see, for instance, [19] for applications of the real-valued case of the estimates of the Bohnenblust-Hille inequality and [7. 12. for applications of the complex-valued case).

One of the most for reaching generalizations of the Hardy-Littlewood inequality is the following theorem (see also [25]):

Theorem 1.1. (See Albuquerque, Araujo, Núñez, Pellegrino and Rueda [1]) Let $m \geq 2$ be a positive integer, $1 \leq k \leq m$ and $n_{1}, \ldots, n_{k} \geq 1$ be positive integers such that $n_{1}+\cdots+n_{k}=m$. If $q_{1}, \ldots, q_{k} \in\left[\frac{1}{1-\left(\frac{1}{p_{1}}+\cdots+\frac{1}{p_{m}}\right)}, 2\right]$ and $0 \leq \frac{1}{p_{1}}+\cdots+\frac{1}{p_{m}} \leq \frac{1}{2}$, then the following assertions are equivalent:

(a) There is a constant $C_{k}=C\left(k, p_{1}, \ldots, p_{m}, q_{1}, \ldots, q_{k}\right)$ such that

$$
\left(\sum_{i_{1}=1}^{\infty}\left(\ldots\left(\sum_{i_{k}=1}^{\infty}\left|T\left(e_{i_{1}}^{n_{1}}, \ldots, e_{i_{k}}^{n_{k}}\right)\right|^{q_{k}}\right)^{\frac{q_{k-1}}{q_{k}}} \ldots\right)^{\frac{q_{1}}{q_{2}}}\right)^{\frac{1}{q_{1}}} \leq C_{k}\|T\|
$$

for all continuous $m$-linear forms $T: \ell_{p_{1}} \times \cdots \times \ell_{p_{m}} \rightarrow \mathbb{R}$.

(b) The numbers $q_{1}, \ldots, q_{k}$ satisfy

$$
\frac{1}{q_{1}}+\cdots+\frac{1}{q_{k}} \leq \frac{k+1}{2}-\left(\frac{1}{p_{1}}+\cdots+\frac{1}{p_{m}}\right)
$$

Above, the notation $e_{j}^{n_{j}}$ represents the $n_{j}$-tuple $\left(e_{j}, \ldots, e_{j}\right)$. The optimal constants of the previous inequalities are essentially unknown. Recent works have shown that in general these constants have a sublinear growth (see [5. 6. 7], and references therein). One of the few cases in which the optimal constants are known for all $m$ is the case of mixed $\left(\ell_{1}, \ell_{2}\right)$-Littlewood inequality (see [21]):

- The optimal constants $C_{(m), \infty}$ satisfying

$$
\sum_{i_{1}=1}^{\infty}\left(\sum_{i_{2}, \ldots, i_{m}=1}^{\infty}\left|T\left(e_{i_{1}}, \ldots, e_{i_{m}}\right)\right|^{2}\right)^{\frac{1}{2}} \leq C_{(m), \infty}\|T\|
$$

for all continuous real $m$-linear forms $T: c_{0} \times \cdots \times c_{0} \rightarrow \mathbb{R}$ are $2^{\frac{m-1}{2}}$.

From now on $p_{0} \approx 1.84742$ is the unique real number satisfying

$$
\Gamma\left(\frac{p_{0}+1}{2}\right)=\frac{\sqrt{\pi}}{2} .
$$

Our main result provides the optimal constants of a Hardy-Littlewood-type inequality that encompasses (4); as far as we know this is the first time in which a Hardy-Littlewood type inequality (except for the case of mixed $\left(\ell_{1}, \ell_{2}\right)$-Littlewood inequality) is proved to have optimal constants with exponential growth:

Theorem 1.2. Let $m \geq 2$ be a positive integer and $p \geq \frac{p_{0}}{p_{0}-1} \approx 2.18006$. The optimal constant $C_{(m), p}$ such that

$$
\left(\sum_{i_{1}=1}^{\infty}\left(\sum_{i_{2}, \ldots, i_{m}=1}^{\infty}\left|T\left(e_{i_{1}}, \ldots, e_{i_{m}}\right)\right|^{2}\right)^{\frac{1}{2} \frac{p}{p-1}} \leq C_{(m), p}\|T\|,\right.
$$

for all continuous m-linear forms $T: \ell_{p} \times c_{0} \times \cdots \times c_{0} \rightarrow \mathbb{R}$ is $\left(2^{\frac{1}{2}-\frac{1}{p}}\right)^{m-1}$.

Note that the above Hardy-Littlewood type inequality holds for $p \geq 2$ (see Theorem 1.1 ). When $p=2$ it is simple to prove that the optimal constants are $C_{(m), p}=1$. As a consequence of the arguments of our proof of Theorem 1.2 we remark that for $2<p<\frac{p_{0}}{p_{0}-1}$ the optimal constants still have exponential growth; so an eventual decrease on the order of the growth when $p \rightarrow 2$ does not happen. Moreover, for $2<p<\frac{p_{0}}{p_{0}-1} \approx 2.18006$, the difference between the bases in the exponential upper and lower estimates of $C_{(m), p}$ is not bigger than $4 \cdot 10^{-4}$ (see the figures 1 and 2 . 
In the final section we also provide upper and lower estimates for the sharp constants $C_{p, \infty}$ of the real case of (2), showing that

$$
2^{\frac{1}{2}-\frac{1}{p}} \leq C_{p, \infty} \leq 2^{\frac{1}{2}-\frac{1}{2 p}}
$$

for all $p \geq \frac{p_{0}}{p_{0}-1} \approx 2.18006$. This result recovers, in particular, the optimality of the constant $\sqrt{2}$ of the real case of the Littlewood's $4 / 3$ inequality obtained in [15].

\section{The Proof of Theorem 1.2}

The Khinchine inequality (see [13]) asserts that, for any $0<q<\infty$, there are positive constants $A_{q}, B_{q}$ such that regardless of the scalar sequence $\left(a_{j}\right)_{j=1}^{n}$ we have

$$
A_{q}\left(\sum_{j=1}^{n}\left|a_{j}\right|^{2}\right)^{\frac{1}{2}} \leq\left(\int_{0}^{1}\left|\sum_{j=1}^{n} a_{j} r_{j}(t)\right|^{q} d t\right)^{\frac{1}{q}} \leq B_{q}\left(\sum_{j=1}^{n}\left|a_{j}\right|^{2}\right)^{\frac{1}{2}},
$$

where $r_{j}$ are the Rademacher functions. For real scalars, U. Haagerup [16] proved that if $p_{0}$ is the number defined in (5) then

$$
A_{q}=\sqrt{2}\left(\frac{\Gamma\left(\frac{q+1}{2}\right)}{\sqrt{\pi}}\right)^{\frac{1}{q}}, \quad \text { for } 1.84742 \approx p_{0}<q<2
$$

and

$$
A_{q}=2^{\frac{1}{2}-\frac{1}{q}}, \quad \text { for } 1 \leq q \leq p_{0} \approx 1.84742 .
$$

Let $T: \ell_{p} \times c_{0} \times \cdots \times c_{0} \rightarrow \mathbb{R}$ be a continuous $m$-linear form. By the Khinchine inequality for multiple sums (see [22]) we know that

$$
\begin{aligned}
& \left(\sum_{i_{1}=1}^{\infty}\left(\sum_{i_{2}, \ldots, i_{m}=1}^{\infty}\left|T\left(e_{i_{1}}, \ldots, e_{i_{m}}\right)\right|^{2}\right)^{\frac{1}{2} \frac{p}{p-1}}\right)^{\frac{p-1}{p}} \\
& \leq\left(A_{\frac{p}{p-1}}^{-1}\right)^{m-1}\left(\sum_{i_{1}=1}^{\infty} \int_{[0,1]^{m-1}}\left|\sum_{i_{2}, \ldots, i_{m}}^{\infty} r_{i_{2}}\left(t_{2}\right) \cdots r_{i_{m}}\left(t_{m}\right) T\left(e_{i_{1}}, \ldots, e_{i_{m}}\right)\right|^{\frac{p}{p-1}} d t_{2} \cdots d t_{m}\right)^{\frac{p-1}{p}} \\
& =\left(A_{\frac{p}{p-1}}^{-1}\right)^{m-1}\left(\int_{[0,1]^{m-1}} \sum_{i_{1}=1}^{\infty}\left|T\left(e_{i_{1}}, \sum_{i_{2}=1}^{\infty} r_{i_{2}}\left(t_{2}\right) e_{i_{2}}, \ldots, \sum_{i_{m}=1}^{\infty} r_{i_{m}}\left(t_{m}\right) e_{i_{m}}\right)\right|^{\frac{p}{p-1}} d t_{2} \cdots d t_{m}\right)^{\frac{p-1}{p}} \\
& \leq\left(A_{\frac{p}{p-1}}^{-1}\right)^{m-1}\left(\int_{[0,1]^{m-1}}\left\|T\left(\cdot \sum_{i_{2}=1}^{\infty} r_{i_{2}}\left(t_{2}\right) e_{i_{2}}, \ldots, \sum_{i_{m}=1}^{\infty} r_{i_{m}}\left(t_{m}\right) e_{i_{m}}\right)\right\|^{\frac{p}{p-1}} d t_{2} \cdots d t_{m}\right)^{\frac{p-1}{p}} \\
& \leq\left(A_{\frac{p}{p-1}}^{-1}\right)^{m-1} \sup _{t_{2}, \ldots, t_{m} \in[0,1]}\left\|T\left(\cdot, \sum_{i_{2}=1}^{\infty} r_{i_{2}}\left(t_{2}\right) e_{i_{2}}, \ldots, \sum_{i_{m}=1}^{\infty} r_{i_{m}}\left(t_{m}\right) e_{i_{m}}\right)\right\| \\
& \leq\left(A_{\frac{p}{p-1}}^{-1}\right)^{m-1}\|T\|=\left(2^{\frac{1}{2}-\frac{1}{p}}\right)^{m-1}\|T\|
\end{aligned}
$$

whenever $p \geq \frac{p_{0}}{p_{0}-1} \approx 2.18006$. Now let us show that $\left(2^{\frac{1}{2}-\frac{1}{p}}\right)^{m-1}$ is the best possible constant. Let $T_{2}: \ell_{p}^{2} \times \ell_{\infty}^{2} \rightarrow \mathbb{R}$ and $T_{2}^{x_{2}}: \ell_{p}^{2} \rightarrow \mathbb{R}$ be given by

$$
T_{2}\left(x_{1}, x_{2}\right)=\left(x_{2}^{1}+x_{2}^{2}\right) x_{1}^{1}+\left(x_{2}^{1}-x_{2}^{2}\right) x_{1}^{2},
$$

and

for each $x_{2} \in \ell_{\infty}^{2}$. Observe that

$$
T_{2}^{x_{2}}\left(x_{1}\right)=T_{2}\left(x_{1}, x_{2}\right),
$$

$$
\left\|T_{2}\right\|=\sup \left\{\left\|T_{2}^{x_{2}}\right\|:\left\|x_{2}\right\|_{\ell_{\infty}^{2}}=1\right\} .
$$


Let us estimate 8. Since $\left(\ell_{p}\right)^{*}=\ell_{\frac{p}{p-1}}$, we have

$$
\begin{aligned}
\left\|T_{2}\right\| & =\sup \left\{\left\|T_{2}^{x_{2}}\right\|:\left\|x_{2}\right\|_{\ell_{\infty}^{2}}=1\right\} \\
& =\sup \left\{\sup _{x_{1} \in B_{\ell_{p}^{2}}}\left|T_{2}^{x_{2}}\left(x_{1}\right)\right|:\left\|x_{2}\right\|_{\ell_{\infty}^{2}}=1\right\} \\
& =\sup \left\{\sup _{x_{1} \in B_{\ell_{p}^{2}}}\left|\left(x_{2}^{1}+x_{2}^{2}\right) x_{1}^{1}+\left(x_{2}^{1}-x_{2}^{2}\right) x_{1}^{2}\right|:\left\|x_{2}\right\|_{\ell_{\infty}^{2}}=1\right\} \\
& =\sup \left\{\left\|\left(x_{2}^{1}+x_{2}^{2}, x_{2}^{1}-x_{2}^{2}, 0,0, \ldots\right)\right\|_{\frac{p}{p-1}}:\left\|x_{2}\right\|_{\ell_{\infty}^{2}}=1\right\} \\
& =\sup \left\{\left(|1+x|^{\frac{p}{p-1}}+|1-x|^{\frac{p}{p-1}}\right)^{\frac{1}{p-1}}: x \in[-1,1]\right\}=2 .
\end{aligned}
$$

In order to verify the last equality, note that since

$$
\sup \left\{\left(|1+x|^{1}+|1-x|^{1}\right)^{1} ; x \in[-1,1]\right\}=2,
$$

by the norm inclusion $\ell_{1} \subset \ell_{\frac{p}{p-1}}$ for $p \in[2, \infty)$, we have $\|\cdot\|_{\ell_{\frac{p}{p-1}}} \leq\|\cdot\|_{\ell_{1}}$. Therefore, for $p \in[2, \infty)$ we have

$$
\sup \left\{\left(|1+x|^{\frac{p}{p-1}}+|1-x|^{\frac{p}{p-1}}\right)^{\frac{p-1}{p}} ; x \in[-1,1]\right\} \leq \sup \left\{\left(|1+x|^{1}+|1-x|^{1}\right)^{1} ; x \in[-1,1]\right\}=2 .
$$

On the other hand, it is obvious that

$$
\sup \left\{\left(|1+x|^{\frac{p}{p-1}}+|1-x|^{\frac{p}{p-1}}\right)^{\frac{p-1}{p}} ; x \in[-1,1]\right\} \geq\left(|1+1|^{\frac{p}{p-1}}+|1-1|^{\frac{p}{p-1}}\right)^{\frac{p-1}{p}}=2 .
$$

In order to show that $\left(2^{\frac{1}{2}-\frac{1}{p}}\right)^{m-1}$ is the best possible constant satisfying (6), let $T_{2}$ be as in 77 and define for all $m \geq 3$ the $m$-linear operator $T_{m}: \ell_{p}^{2^{m-1}} \times \ell_{\infty}^{2^{m-1}} \times \cdots \times \ell_{\infty}^{2^{m-1}} \rightarrow \mathbb{R}$ by

$$
\begin{aligned}
T_{m}\left(x_{1}, \ldots, x_{m}\right)= & \left(x_{m}^{1}+x_{m}^{2}\right) T_{m-1}\left(x_{1}, \ldots, x_{m-1}\right) \\
& +\left(x_{m}^{1}-x_{m}^{2}\right) T_{m-1}\left(S_{p}^{2^{m-2}}\left(x_{1}\right), S_{0}^{2^{m-2}}\left(x_{2}\right), S_{0}^{2^{m-3}}\left(x_{3}\right) \ldots, S_{0}^{2}\left(x_{m-1}\right)\right),
\end{aligned}
$$

where $x_{1} \in \ell_{p}^{2^{m-1}}, x_{k} \in \ell_{\infty}^{2^{m-1}}$ for all $k=2, \ldots, m$, and $S_{p}: \ell_{p} \rightarrow \ell_{p}$ and $S_{0}: c_{0} \rightarrow c_{0}$ are the backward shifts. By induction on $m \geq 2$ we shall show that

$$
\left\|T_{m}\right\|=2^{m-1} .
$$

The case $m=2$ is already done in 9 . Let us suppose that $\left\|T_{m-1}\right\|=2^{(m-1)-1}$. Therefore,

$$
\begin{aligned}
\left|T_{m}\left(x_{1}, \ldots, x_{m}\right)\right| \leq & \left|x_{m}^{1}+x_{m}^{2} \| T_{m-1}\left(x_{1}, \ldots, x_{m-1}\right)\right| \\
& +\left|x_{m}^{1}-x_{m}^{2}\right|\left|T_{m-1}\left(S_{p}^{2^{m-2}}\left(x_{1}\right), S_{0}^{2^{m-2}}\left(x_{2}\right), S_{0}^{2^{m-3}}\left(x_{3}\right) \ldots, S_{0}^{2}\left(x_{m-1}\right)\right)\right| \\
\leq & 2^{m-2}\left[\left|x_{m}^{1}+x_{m}^{2}\right|\left\|x_{1}\right\|_{\ell_{p}^{2 m-1}} \cdots\left\|x_{m-1}\right\|_{\ell_{\infty}^{2 m-1}}\right. \\
& \left.+\left|x_{m}^{1}-x_{m}^{2}\right|\left\|S_{p}^{2^{m-2}}\left(x_{1}\right)\right\|_{\ell_{p}^{2 m-1}}\left\|S_{0}^{2^{m-2}}\left(x_{2}\right)\right\|_{\ell_{\infty}^{2 m-1}}\left\|S_{0}^{2^{m-3}}\left(x_{3}\right)\right\|_{\ell_{\infty}^{2 m-1}} \cdots\left\|S_{0}^{2}\left(x_{m-1}\right)\right\|_{\ell_{\infty}^{2}}\right] \\
\leq & 2^{m-2}\left[\left|x_{m}^{1}+x_{m}^{2}\right|+\left|x_{m}^{1}-x_{m}^{2}\right|\right]\left\|x_{1}\right\|_{\ell_{p}^{2 m-1}} \cdots\left\|x_{m-1}\right\|_{\ell_{\infty}^{2^{m}-1}} \\
= & 2^{m-1}\left\|x_{1}\right\|_{\ell_{p}^{2 m-1}} \cdots\left\|x_{m-1}\right\|_{\ell_{\infty}^{2 m-1}} \max \left\{\left|x_{m}^{1}\right|,\left|x_{m}^{2}\right|\right\} \\
\leq & 2^{m-1}\left\|x_{1}\right\|_{\ell_{p}^{2 m-1}} \cdots\left\|x_{m}\right\|_{\ell_{\infty}^{2 m-1}} .
\end{aligned}
$$

We thus have $\left\|T_{m}\right\| \leq 2^{m-1}$. Now consider $a_{m}=e_{1}+e_{2}$ and note that

$$
\begin{aligned}
\left\|T_{m}\right\| & \geq \sup \left\{\left|T_{m}\left(x_{1}, \ldots, x_{m-1}, a_{m}\right)\right|: x_{1} \in B_{\ell_{p}^{2 m-1}}, x_{2} \in B_{\ell_{\infty}^{2 m-1}}, \ldots, x_{m-1} \in B_{\ell_{\infty}^{2 m-1}}\right\} \\
& =2\left\|T_{m-1}\right\|=2^{m-1}
\end{aligned}
$$

and hence $\left\|T_{m}\right\|=2^{m-1}$. 
Since

the proof is done.

$$
\frac{\left(\sum_{i_{1}}\left(\sum_{i_{2}, \ldots, i_{m}}\left|T_{m}\left(e_{i_{1}, \ldots,} e_{i_{m}}\right)\right|^{2}\right)^{\frac{1}{2} \frac{p}{p-1}}\right)^{\frac{p-1}{p}}}{\left\|T_{m}\right\|}=\left(2^{\frac{1}{2}-\frac{1}{p}}\right)^{m-1}
$$

\section{Final REMARKS}

The same argument used in the proof of Theorem 1.2 shows that for $2<p<\frac{p_{0}}{p_{0}-1} \approx 2.18006$ the optimal constants also have exponential growth; curiously, for $p=2$ the situation is quite different and the optimal constants are 1 . In fact, note that the second part of the proof (the optimality proof) holds for all $p \geq 2$. Moreover, the first part of the proof gives us the estimate $C_{(m), p} \leq\left(A_{\frac{p}{p-1}}^{-1}\right)^{m-1}$. We thus have, for $2 \leq p<\frac{p_{0}}{p_{0}-1} \approx 2.18006$, the following inequalities

$$
\left(2^{\frac{1}{2}-\frac{1}{p}}\right)^{m-1} \leq C_{(m), p} \leq\left(\frac{1}{\sqrt{2}}\left(\frac{\Gamma\left(\frac{2 p-1}{2 p-2}\right)}{\sqrt{\pi}}\right)^{\frac{1-p}{p}}\right)^{m-1} .
$$

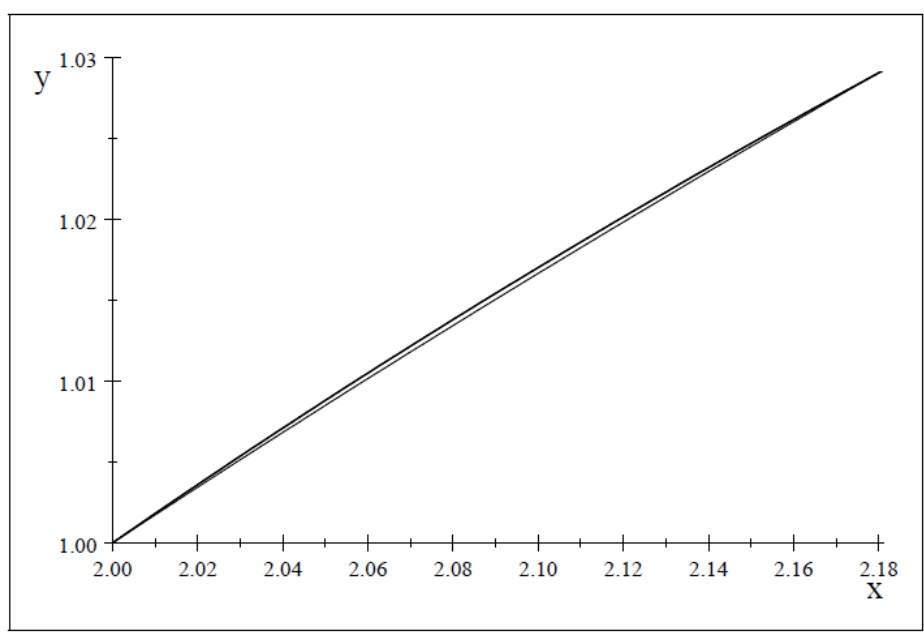

FIGURE 1. Plots of the functions $A_{\frac{x}{x-1}}^{-1}$ and $2^{\frac{1}{2}-\frac{1}{x}}$, for $x \in\left[2, \frac{p_{0}}{p_{0}-1}\right]$

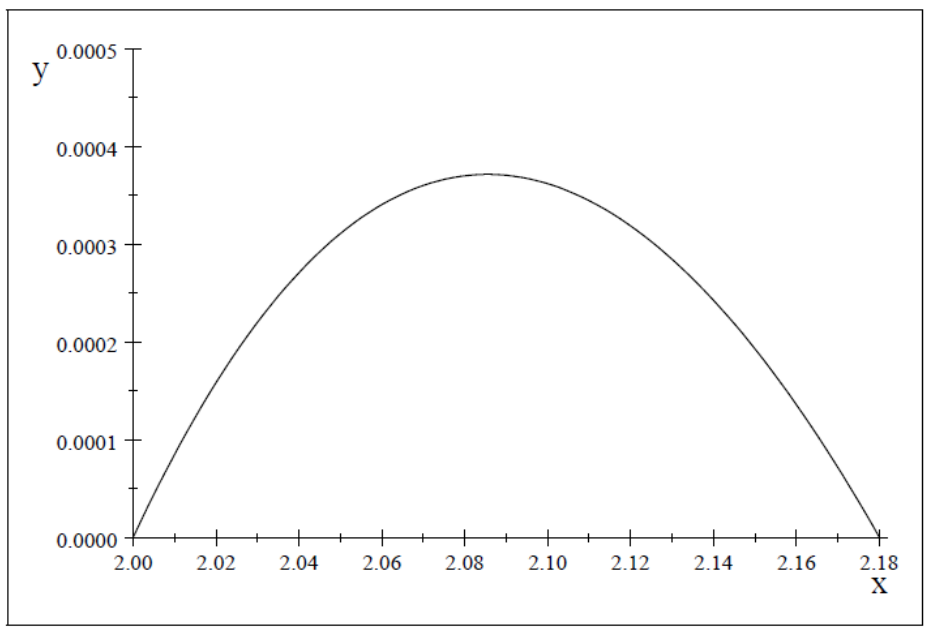

Figure 2. Plot of the function $\left(A_{\frac{x}{x-1}}^{-1}-2^{\frac{1}{2}-\frac{1}{x}}\right)$, for $x \in\left[2, \frac{p_{0}}{p_{0}-1}\right]$ 
For $p \geq 2$, we know that

$$
\left(\sum_{j=1}^{\infty}\left(\sum_{k=1}^{\infty}\left|A\left(e_{j}, e_{k}\right)\right|^{\lambda}\right)^{\frac{1}{\lambda} 2} \leq \sqrt{2}\|A\|\right.
$$

with $\lambda=\frac{p}{p-1}$, for all continuous bilinear forms $A: \ell_{p} \times c_{0} \rightarrow \mathbb{R}$ (see, for instance, 2, Theorem 1.2 and Remark 5.1]). By interpolating (10) and the result of Theorem 1.2 for $m=2$ in the sense of [2] or using the Hölder inequality for mixed sums ([8]) we obtain, for $p \geq \frac{p_{0}}{p_{0}-1} \approx 2.18006$,

$$
\left(\sum_{j, k=1}^{\infty}\left|A\left(e_{j}, e_{k}\right)\right|^{\frac{4 p}{3 p-2}}\right)^{\frac{3 p-2}{4 p}} \leq(\sqrt{2}\|A\|)^{1 / 2}\left(2^{\frac{p-2}{2 p}}\|A\|\right)^{1 / 2}=2^{\frac{1}{2}-\frac{1}{2 p}}\|A\| .
$$

Using the approach of the previous section we obtain the lower estimate

$$
C_{p, \infty} \geq \frac{\left(\sum_{j, k=1}^{2}\left|T_{2}\left(e_{j}, e_{k}\right)\right|^{\frac{4 p}{3 p-2}}\right)^{\frac{3 p-2}{4 p}}}{\left\|T_{2}\right\|}=\frac{4^{\frac{3 p-2}{4 p}}}{2}=2^{\frac{1}{2}-\frac{1}{p}}
$$

and thus

$$
2^{\frac{1}{2}-\frac{1}{p}} \leq C_{p, \infty} \leq 2^{\frac{1}{2}-\frac{1}{2 p}}
$$

When $p=\infty$ we recover the well known optimal estimate of the famous Littlewood's $4 / 3$ that can be found in [15].

Acknowledgement. The authors are indebted to the two anonymous referees for their important contributions to the final version of this paper.

\section{REFERENCES}

[1] N. Albuquerque, G. Araújo, D. Núñez-Alarcón, D. Pellegrino, and P. Rueda, Bohnenblust-Hille and Hardy-Littlewood inequalities by blocks, arXiv:1409.6769v6 [math.FA].

[2] N. Albuquerque, F. Bayart, D. Pellegrino, J. Seoane-Sepulveda, Sharp generalizations of the multilinear Bohnenblust-Hille inequality, J. Funct. Anal. 266 (2014), no. 6, 3726-3740.

[3] N. Albuquerque, F. Bayart, D. Pellegrino and J. B. Seoane-Sepúlveda, Optimal Hardy-Littlewood type inequalities for polynomials and multilinear operators, Isr. J. Math. 211 (2016), 197-220.

[4] G. Araújo, D. Pellegrino, Lower bounds for the constants of the Hardy-Littlewood inequalities, Linear Algebra Appl. 463 (2014), $10-15$.

[5] G. Araújo, D. Pellegrino, On the constants of the Bohnenblust-Hille and Hardy-Littlewood inequalities, to appear in Bull. Braz. Math. Soc.

[6] G. Araújo, D. Pellegrino, D. Diniz P. Silva e Silva, On the upper bounds for the constants of the Hardy-Littlewood inequality, J. Funct. Anal. 267 (2014), no. 6, 1878-1888.

[7] F. Bayart, D. Pellegrino and J. B. Seoane-Sepúlveda, The Bohr radius of the $n$-dimensional polydisk is equivalent to $\sqrt{(\log n) / n}$, Adv. Math. 264 (2014), 726-746.

[8] A. Benedek, R. Panzone, The space $L_{p}$, with mixed norm, Duke Math. J. 281961 301-324.

[9] H. F. Bohnenblust, E. Hille, On the absolute convergence of Dirichlet series, Ann. of Math. 32 (1931), 600-622.

[10] G. Botelho, J. Santos, A Pietsch domination theorem for $\left(\ell_{p}^{s}, \ell_{p}\right)$-summing operators, Arch. Math. (Basel) 104 (2015), $47-52$.

[11] W. Cavalcante, D. Núñez-Alarcón, Remarks on the Hardy-Littlewood inequality for $m$-homogeneous polynomials and $m$-linear forms, to appear in Quaest. Math.

[12] A. Defant, L. Frerick, J. Ortega-Cerdá, M. Ounaïes, K. Seip, The Bohnenblust-Hille inequality for homogeneous polynomials is hypercontractive, Ann. of Math. (2), 174 (2011), 485-497.

[13] J. Diestel, H. Jarchow, A. Tonge, Absolutely summing operators, Cambridge University Press, Cambridge, 1995.

[14] V. Dimant and P. Sevilla-Peris, Summation of coefficients of polynomials on $\ell_{p}$ spaces, Publ. Mat. 60 (2016), $289-310$.

[15] D. Diniz, G. Muñoz-Fernández, D. Pellegrino, J. Seoane-Sepúlveda, Lower bounds for the constants in the Bohnenblust-Hille inequality: the case of real scalars, Proc. Amer. math. Soc. 142 (2014), 575-580.

[16] U. Haagerup, The best constants in the Khinchine inequality, Studia Math. 70 (1982) 231-283.

[17] G. Hardy and J. E. Littlewood, Bilinear forms bounded in space [p, q], Quart. J. Math. 5 (1934), 241-254.

[18] J. E. Littlewood, On bounded bilinear forms in an infinite number of variables, Quart. J. Math. 1 (1930), 164-174.

[19] A. Montanaro, Some applications of hypercontractive inequalities in quantum information theory. J. Math. Phys. 53 (2012), no. $12,122206,15$ pp.

[20] B. Osikiewicz, A. Tonge, An interpolation approach to Hardy-Littlewood inequalities for norms of operators on sequence spaces, Linear Algebra Appl. 331 (2001), 1-9.

[21] D. Pellegrino, The optimal constants of the mixed $\left(\ell_{1}, \ell_{2}\right)$-Littlewood inequality, J. Number Theory 160 (2016), 11-18.

[22] D. Popa, Multiple Rademacher means and their applications, J. Math. Anal. Appl. 386 (2012), 699-708.

[23] D. Popa, Multiple summing operators on $\ell_{p}$-spaces, Studia Math. 225 (2014), no. 1, 9-28.

[24] P. Rueda, E.A. Sánchez-Pérez, Factorization of $p$-dominated polynomials through $L_{p}$-spaces, Michigan Math. J. 63 (2014), no. 2, 345-353. 
[25] J. Santos and T. Velanga, A note on the Bohnenblust-Hille inequality for multilinear forms, arXiv:1604.00040 2 [math.FA].

[26] D. M. Serrano-Rodríguez, Improving the closed formula for subpolynomial constants in the multilinear Bohnenblust-Hille inequalities, Linear Algebra Appl. 438 (2013) 3124-3138.

(T. Nogueira) Departamento de Matemática, Universidade Federal da Paraíba, 58.051-900 - João Pessoa, Brazil. E-mail address: tonykleverson@gmail.com

(D. Núñez-Alarcón) Departamento de Matemática, Universidade Federal de Pernambuco, 50.740-560 - Recife, Brazil, \& Department of Mathematical Sciences, Kent State University, Kent, Ohio 44242, USA

E-mail address: danielnunezal@gmail.com

(D. Pellegrino) Departamento de Matemática, Universidade Federal da Paraíba, 58.051-900 - João Pessoa, Brazil.

E-mail address: pellegrino@pq.cnpq.br and dmpellegrino@gmail.com 